\title{
An Adaptive Wavelet Viscosity Method for Hyperbolic Conservation Laws
}

\author{
Daniel Castaño Díez, ${ }^{1}$ Max Gunzburger, ${ }^{2}$ Angela Kunoth ${ }^{3}$ \\ ${ }^{1}$ EMBL Heidelberg, Meyerhofstraße 1, 69117 Heidelberg, Germany \\ ${ }^{2}$ School of Computational Science, Florida State University, Tallahassee Florida \\ 32306-4120 \\ ${ }^{3}$ Institut für Angewandte Mathematik und Institut für Numerische Simulation, Universität \\ Bonn, Wegelerstr. 6, 53115 Bonn, Germany
}

Received 9 March 2007; accepted 23 October 2007

Published online 18 March 2008 in Wiley InterScience (www.interscience.wiley.com).

DOI 10.1002/num.20322

We extend the multiscale finite element viscosity method for hyperbolic conservation laws developed in terms of hierarchical finite element bases to a (pre-orthogonal spline-)wavelet basis. Depending on an appropriate error criterion, the multiscale framework allows for a controlled adaptive resolution of discontinuities of the solution. The nonlinearity in the weak form is treated by solving a least-squares data fitting problem. C) 2008 Wiley Periodicals, Inc. Numer Methods Partial Differential Eq 24: 1388-1404, 2008

Keywords: wavelet methods, hyperbolic conservation laws, adaptive methods

\section{INTRODUCTION}

Consider Burgers' equation with periodic boundary conditions and given initial condition $g$,

$$
\begin{aligned}
\frac{\partial u}{\partial t}+\frac{\partial}{\partial x}\left(\frac{u^{2}}{2}\right) & =0 & & \text { on } \Omega_{T}:=(0,1) \times(0, T), \\
u(0, t) & =u(1, t) & & \text { in }(0, T), \\
u(x, 0) & =g(x) & & \text { in }(a, b) \subset \mathbb{R},
\end{aligned}
$$

which was introduced as a simplified model for inviscid fluid flow. Because of the nonlinearity, its solution is known to develop isolated discontinuities after finite time.

Discretization schemes for the Burgers' equation based on the classical type of weak formulations that are standard for elliptic partial differential equations (PDEs) cause strong numerical instabilities. One can stabilize such methods by adding an artificial diffusion term to the equation. However, this procedure usually results in a "smearing out" of problem-inherent discontinuities

Correspondence to: Max Gunzburger, School of Computational Science, Florida State University, Tallahassee, FL 32306-4120 (e-mail: gunzburg@scs.fsu.edu)

Contract grant sponsor: Deutsche Forschungsgemeinschaft; contract grant number: KU 1028/7-1

Contract grant sponsor: Universität Bonn; contract grant number: SFB 611

Contract grant sponsor: U.S. National Science Foundation; contract grant number: DMS-0308845

(c) 2008 Wiley Periodicals, Inc. 
and leads to a great sacrifice in accuracy, even in regions where the solution is smooth. Much better results are attained by spectral viscosity methods in which diffusion is added only to the highest frequencies; see [1] and the series of articles that followed. The method was extended in [3] to a multiscale finite element formalism in terms of hierarchical finite element bases [2]. In the spirit of spectral viscosity methods, artificial diffusion is added only at the highest hierarchical levels. Convergence to the entropy solution was proved for the scalar univariate case. A discussion about the efficient implementation of the multiscale finite element viscosity method and examples in two space dimensions can be found in [4]. Moreover, it was demonstrated numerically in [3-5] that, in practice, regularization can be restricted to the highest hierarchical level.

The results in [3-5] were obtained employing hierarchical finite element bases with respect to a uniform grid. Here, we adapt the formulation of $[3,5]$ to construct a converging wavelet-based method to numerically solve Burgers' equation. We will then apply an elementary smoothing on the solution based on elementary properties of a wavelet-specific detection of singularities.

We note that a similar frequency-dependent artificial viscosity method was used introduced in [6] for slightly viscous, convection dominated problems and further developed and analyzed in [7].

Adaptive schemes that are capable of concentrating degrees of freedom in the neighborhood of discontinuities provide a particular high potential for efficient numerical solution. Thus, after studying the quality attainable in the wavelet formulation with respect to a uniform grid, we will turn our attention to its efficiency by proposing and testing an adaptive algorithm that concentrates the degrees of freedom only in the neighborhood of singularities, following them as they evolves with time. Specifically, diffusion is added only in the vicinity of discontinuities.

We describe our scheme in terms of a class of piecewise linear continuous spline-wavelets. We note that, in principle, an extension to higher-order spline-wavelets is possible, but, of course, at the expense of a corresponding larger support.

We also note that different types of adaptive wavelet methods for solving time-dependent PDEs have been developed in the past; see, e.g., [8]. For the treatment of shocks, see, in particular, [9].

The remainder of the article is organized as follows. In the next section, we describe the class of spline-wavelets used here. This is followed in Section III by the description of a wavelet viscosity method together with convergence results. In Section IV, we study, through numerical experimentation, how singularities can be easily detected by wavelets; this should be contrasted to the case of spectral methods for which such detection is much more difficult. Section V describes an adaptive wavelet viscosity scheme, adding viscosity only in the vicinity of a discontinuity; in this way, selective application of artificial diffusion is effected with respect to both the frequency and spatial domains. Finally, in Section VI, we describe how nonlinearities are evaluated in our scheme by means of a least-squares approach developed in [10] in the context of scattered data fitting problems.

Throughout this article, we use the relation $A \sim B$ to express $A \lesssim B$ and $A \gtrsim B$, which means that $A$ can be estimated from above and below by a constant multiple of $B$, independent of all parameters on which $A$ or $B$ may depend.

\section{SOME BASICS ABOUT WAVELETS}

Fundamental to the method described below are appropriate wavelet bases for a function space $H \subseteq L_{2}(\Omega)$ living on a bounded domain $\Omega \subset \mathbb{R}^{d}$ and possessing certain properties essential for the efficient numerical solution of PDEs; see, e.g., [11,12] and references therein for such constructions. Wavelets are indexed by a parameter $\lambda=(j, k, \mathbf{e})$ which encodes information such 
as the resolution level or scale $|\lambda|:=j$, the spatial location $k$, and possibly the type $\mathbf{e}$ of wavelet in the multivariate case. For univariate domains $\Omega=(a, b)$, the indices are simply of the form $\lambda=(j, k)$. In view of the finite domain $\Omega$, there is a coarsest level $j_{0}$. We denote the infinite set of all possible indices by II. A wavelet basis is a collection of functions

$$
\Psi:=\left\{\psi_{\lambda}: \lambda \in I I\right\} \subset H
$$

having the following properties:

(R) $\Psi$ constitutes a Riesz basis for $H$ : every $v \in H$ has a unique expansion in terms of $\Psi$,

$$
v=\sum_{\lambda \in I} v_{\lambda} \psi_{\lambda}, \quad \mathbf{v}:=\left(v_{\lambda}\right)_{\lambda \in I}
$$

and its expansion coefficients satisfy a norm equivalence relation, i.e., there exist constants $0<c_{H} \leq C_{H}<\infty$ such that

$$
c_{H}\|\mathbf{v}\|_{\ell_{2}(I I)} \leq\left\|\sum_{\lambda \in I} v_{\lambda} \psi_{\lambda}\right\|_{H} \leq C_{H}\|\mathbf{v}\|_{\ell_{2}(I I)}, \quad \mathbf{v} \in \ell_{2}(I I),
$$

holds. In other words, wavelet expansions induce isomorphisms between the space of functions $H$ and the sequence space $\ell_{2}(I I)$.

(L) The functions $\psi_{\lambda}$ are local: for each $\lambda \in I$ II one has

$$
\operatorname{diam}\left(\operatorname{supp} \psi_{\lambda}\right) \sim 2^{-|\lambda|} .
$$

(CP) There exists an integer $\tilde{m}$ such that

$$
\left\langle v, \psi_{\lambda}\right\rangle \lesssim 2^{-|\lambda|(d / 2+\widetilde{m})}|v|_{W_{\infty}^{\tilde{m}}\left(\operatorname{supp} \psi_{\lambda}\right)},
$$

where $\langle\cdot, \cdot\rangle$ is the duality pairing between $H$ and its topological dual $H^{\prime}$.

Property (CP) means that integrating a function against a wavelet is like taking an $\tilde{m}$ th order difference which annihilates the smooth part of $v$. This feature is called the cancellation property and comes into play for the evaluation of nonlinearities. Moreover, the underlying moment conditions entail a direct approximation estimate for a finite-dimensional subspace $V_{J}$ that is spanned by all wavelet functions up to a highest refinement level $J$.

Properties (R), (L), and (CP) allow one to prove strong theoretical statements such as (asymptotically) optimal condition number estimates for linear elliptic operators or convergence results for adaptive methods for linear and nonlinear variational problems [12-15]. At the same time, they still allow one to work computationally with piecewise polynomials. Remarks on the concrete constructions of biorthogonal wavelet bases on bounded Euclidean tensor product domains based on B-Splines can be found in [12]. Constructions on multivariate box domains can be handled by tensor products. For more general domains or manifolds, see the constructions in [16] and references cited therein.

Here we employ the piecewise linear boundary-adapted B-spline pre-wavelets from [17] that are a special case of the pre-wavelets on the interval constructed in [18]. These bases have the additional property that they are 
(O) semi-orthogonal with respect to $L_{2}(\Omega)$, that is, for $|\lambda| \neq|\mu|$, one has

$$
\int_{\Omega} \psi_{\lambda}(x) \psi_{\mu}(x) d x=0
$$

Consequently, entries of mass matrices corresponding to wavelets on different refinement levels vanish.

\section{A WAVELET VISCOSITY METHOD}

First, we recall the main result obtained in $[3,5]$ about the convergence of approximations to the solution of (1.1) constructed using uniform grid hierarchical finite element bases. In the monovariate case, the ansatz function of the approach can be written as

$$
u_{J}(x, t)=\sum_{\lambda \in \Lambda_{J}} d_{\lambda}^{\mathrm{hb}}(t) \psi_{\lambda}^{\mathrm{hb}}(x)
$$

where $\left\{\psi_{\lambda}^{\mathrm{hb}}\right\}_{\lambda \in \Lambda_{J}}$ is the hierarchical basis for the space $V_{J}$ of continuous piecewise linear functions on each subinterval $2^{-j}[i, i+1]$ for $i=0, \ldots, 2^{j}-1$.

The hierarchical basis approximation of (1.1) is thus given by a $u_{J}(x, t) \in V_{J}$ such that $u_{J}(\cdot, 0)=g_{J}$ for some projection $g_{J}$ of $g$ onto $V_{J}$ that solves the regularized weak formulation of (1.1) with respect to space

$$
\int_{0}^{1}\left[\frac{\partial u_{J}}{\partial t}+\frac{\partial}{\partial x}\left(\frac{u_{J}^{2}}{2}\right)\right] v d x+v_{J} \int_{0}^{1}\left[\frac{\partial}{\partial x}\left(Q_{J}^{j_{\mathrm{cut}}} u_{J}\right) \frac{\partial v}{\partial x}\right] d x=0
$$

for every $v \in V_{J}$. Here, $v_{J}$ is an artificial viscosity parameter and the operator $Q_{J}^{j_{\text {cut }}}$ eliminates the components whose scale is smaller than $j_{\text {cut }}$, i.e.,

$$
Q_{J}^{j_{\text {cut }}}\left(\sum_{\lambda \in \Lambda_{J}} d_{\lambda}^{\mathrm{hb}} \psi_{\lambda}^{\mathrm{hb}}\right):=\sum_{\lambda \in \Lambda_{J},|\lambda| \geq j_{\mathrm{cut}}} d_{\lambda}^{\mathrm{hb}} \psi_{\lambda}^{\mathrm{hb}}
$$

With these ingredients, we can state the following convergence result. We note that the assumption that the sequence of approximate solutions remains uniformly bounded is also invoked for frequency-dependent artificial viscosity methods in the spectral and finite element methods settings; see [1] and [3], respectively.

Theorem 3.1. [3] Let $\left\{u_{J}\right\}_{J=j_{0}}^{\infty}$ denote a sequence of hierarchical basis approximations determined by (3.2). Assume that $u_{J}$ is uniformly bounded in $L_{\infty}\left(\Omega_{T}\right)$ and that

$$
\begin{aligned}
v_{J} & \rightarrow 0 \quad \text { as } J \rightarrow \infty, \\
v_{J} & \gtrsim 2^{-J}, \\
\sqrt{v_{J}}\left\|\frac{\partial}{\partial x}\left[\left(I-Q_{J}\right) u_{J}\right]\right\|_{L_{2}(0,1)} & \lesssim\left\|u_{J}\right\|_{L_{2}(0,1)},
\end{aligned}
$$




$$
\begin{aligned}
\sqrt{v_{J}}\left\|\frac{\partial}{\partial x}\left[\left(I-Q_{J}\right) \frac{\partial u_{J}}{\partial t}\right]\right\|_{L_{2}(0,1)} & \lesssim\left\|\frac{\partial u_{J}}{\partial t}\right\|_{L_{2}(0,1)}, \\
\left\|\frac{d}{d x}\left(Q_{J} g_{J}\right)\right\|_{L_{2}(0,1)} & \lesssim\left\|\frac{d g_{J}}{d x}\right\|_{L_{2}(0,1)} .
\end{aligned}
$$

Then, there exists a subsequence of $\left\{u_{J}\right\}_{J=j_{0}}^{\infty}$ that converges strongly in $L_{2}\left(\Omega_{T}\right)$ to a solution $u \in L_{2}\left(\Omega_{T}\right)$ of $(1.1)$.

In the full grid approximation, the convergence result expressed in Theorem 3.1 is directly applicable to a wavelet formulation. We expand the solution $u_{J}$ to (3.2) as

$$
u_{J}(x, t)=\sum_{\lambda \in \Lambda_{J}} d_{\lambda}(t) \psi_{\lambda}(x)
$$

where $\left\{\psi_{\lambda}\right\}_{\lambda \in \Lambda_{J}}, \Lambda_{J} \subset I I$, is the linear spline-wavelet basis of $V_{J} \subset L_{2}(a, b)$, consisting of all wavelets $\psi_{\lambda}$ on all levels $j_{0} \leq|\lambda| \leq J$. (With abuse of notation, we use here the same symbol $\Lambda_{J}$ as in (3.1) to denote the corresponding finite-dimensional index set.)

The operator $Q_{J}^{j_{\text {cut }}}$ that kills oscillations on scales smaller than $j_{\text {cut }}$ has the same form as in (3.3),

$$
Q_{J}^{j_{\text {cut }}}\left(\sum_{\lambda \in \Lambda_{J}} d_{\lambda} \psi_{\lambda}(x)\right)=\sum_{\lambda \in \Lambda_{J},|\lambda| \geq j_{\text {cut }}} d_{\lambda} \psi_{\lambda}(x) .
$$

Particularly, we can make a simple choice of parameters that guarantees asymptotic convergence of the (Petrov-)Galerkin method in the wavelet basis.

Proposition 3.2. With the choices $v_{J} \sim 2^{-J}$ and $j_{\text {cut }} \leq \frac{J}{2}$, the assumptions (3.4) to (3.8) for a function $u_{J}$ of the form (3.9) satisfying the regularized weak formulation (3.1) are met.

Proof. The properties for the continuous hierarchical piecewise linear basis $\left\{\psi_{\lambda}^{\mathrm{hb}}\right\}_{\lambda \in \Lambda_{J}}$ established in [3] directly carry over to the case of semi-orthogonal linear spline-wavelets $\left\{\psi_{\lambda}\right\}_{\lambda \in \Lambda_{J}}$ since they provide in the finite-dimensional case on a uniform grid a basis for the same space $V_{J}$.

For convenience, we include alternative and simpler proofs that take advantage of property (R). For all $v \in V_{J}$, we have

$$
\left(I-Q_{J}^{j_{\mathrm{cut}}}\right) v=\sum_{\lambda \in \Lambda,|\lambda|<j_{\mathrm{cut}}} d_{\lambda} \psi_{\lambda}
$$

Since

$$
\sum_{\lambda \in \Lambda,|\lambda|<j_{\text {cut }}}\left|d_{\lambda}\right|^{2} \leq \sum_{\lambda \in \Lambda}\left|d_{\lambda}\right|^{2}
$$

by the Riesz property $\sum_{\lambda \in I}\left|w_{\lambda}\right|^{2} \sim\|w\|_{L_{2}(0,1)}^{2}$ that holds for any $w=\sum_{\lambda \in I} w_{\lambda} \psi_{\lambda}$, we can write

$$
\left\|\left(I-Q_{J}^{j_{\mathrm{cut}}}\right) v\right\|_{L_{2}(0,1)} \lesssim\|v\|_{L_{2}(0,1)} .
$$


Now, $\left(I-Q_{J}^{j_{\text {cut }}}\right) v$ is linear on intervals of length $2^{-\left(j_{\text {cut }}+1\right)}$ so that

$$
\left\|\frac{\partial}{\partial x}\left[\left(I-Q_{J}^{j_{\text {cut }}}\right) v\right]\right\|_{L_{2}(0,1)} \lesssim 2^{j_{\text {cut }}}\left\|\left(I-Q_{J}^{j_{\text {cut }}}\right) v\right\|_{L_{2}(0,1)} .
$$

Multiplying by $2^{-J / 2}$ and using (3.11), we have

$$
2^{-\frac{J}{2}}\left\|\frac{\partial}{\partial x}\left[\left(I-Q_{J}^{j_{\mathrm{cut}}}\right) v\right]\right\|_{L_{2}(0,1)} \lesssim 2^{-\frac{J}{2}+j_{\mathrm{cut}}}\|v\|_{L_{2}(0,1)} .
$$

Since we have chosen $j_{\text {cut }} \leq \frac{J}{2}$, taking $v \in\left\{u_{J}, \frac{\partial u_{J}}{\partial t}\right\}$, conditions (3.6) and (3.7) are satisfied.

Finally, (3.8) is a consequence of the Riesz-Basis property on $H^{1}(0,1)$.

\section{NUMERICAL EXPERIMENTS - THE FULL-GRID CASE}

\section{A. Smoothing of Gibbs Phenomenon}

As a model problem, consider the following form of Burgers' equation (1.1) with a particular set of boundary and smooth initial conditions:

$$
\begin{aligned}
\frac{\partial u}{\partial t}+\frac{\partial}{\partial t}\left(\frac{u^{2}}{2}\right) & =0 & & \text { on }(0,1) \times(0, T), \\
u(0, t) & =u(1, t) & & \text { for all } t \in(0, T), \\
u(x, 0) & =\sin (2 \pi x) & & \text { for } x \in(0,1)
\end{aligned}
$$

that gives rise to a stationary shock. As time increases, the derivative of $u$ grows larger in a decreasing neighborhood of $x=0.5$, eventually developing a sharp jump. After this singularity is formed, the amplitude of the solution starts to decrease.

We have computed an approximate solution using a slight modification, with respect to boundary conditions, of our (preorthogonal spline-)wavelets described earlier. To satisfy the periodicity conditions, we have substituted the boundary adaption with a periodization of the basis ensuring that $\psi_{\lambda}(0)=\psi_{\lambda}(1)$ for all $\lambda \in \Lambda_{J}$. We have used the full grid when we increase the maximal level of resolution $J$ at time $t=0.5$ for which the sharp shock has been formed and is about to start reducing in amplitude.

As expected, numerical instabilities appear if no viscosity is added (see Fig. 1) and the scheme does not converge when only refining the ansatz space. Applying the results from Section III, we
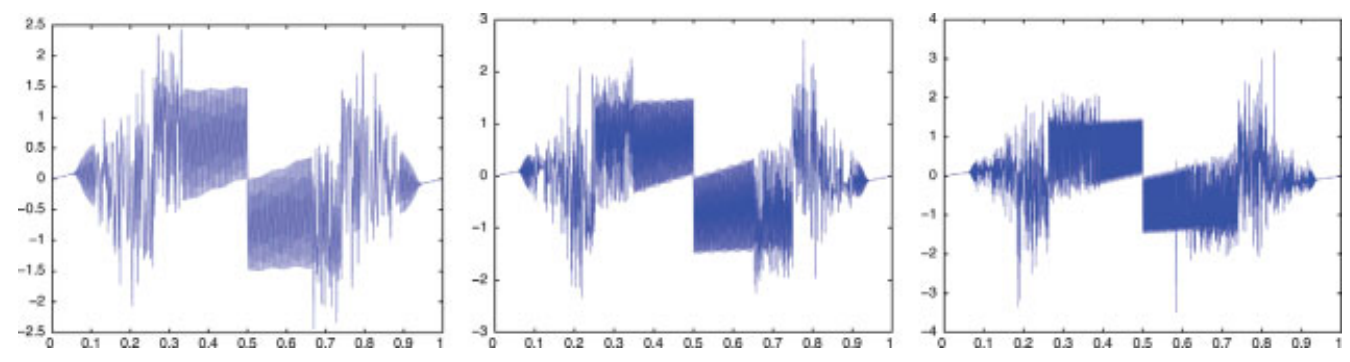

FIG. 1. Wavelet solutions for the stationary shock problem (4.1) using different resolutions $(J=8,9,10$ from left to right) without applying artificial diffusion. [Color figure can be viewed in the online issue, which is available at www.interscience.wiley.com.] 

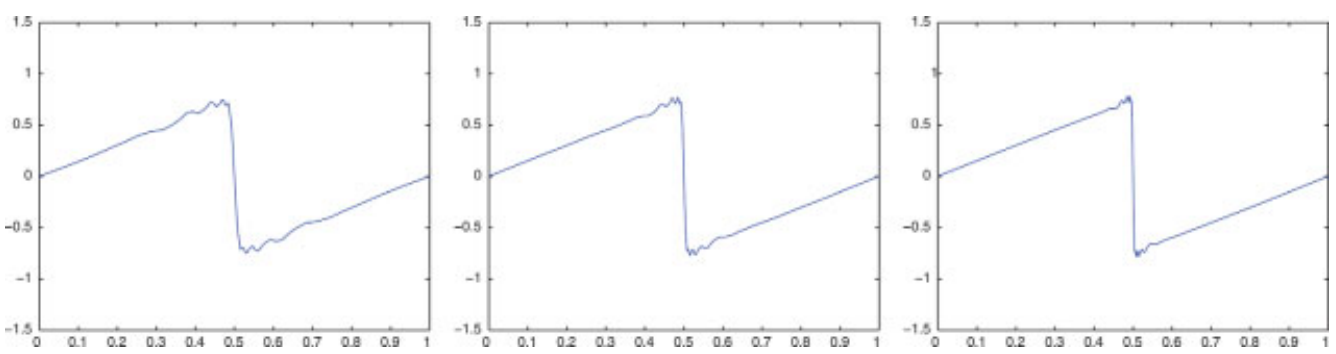

FIG. 2. Wavelet solutions for the stationary shock problem (4.1) using different resolutions. At each resolution $J$, a $j_{\text {cut }}$ that meets the requirements of Theorem 3.1 for convergence of the Galerkin method is applied: from left to right, $j_{\text {cut }}=3$ for $J=8 ; j_{\text {cut }}=4$ for $J=9$, and $j_{\text {cut }}=5$ for $J=10$. [Color figure can be viewed in the online issue, which is available at www.interscience.wiley.com.]

can ensure convergence if viscosity is added at least to the upper $J / 2$ levels of a discretization with a maximal level of resolution $J$. This is confirmed by the results in Fig. 2.

An important point is that the frequency-restricted wavelet viscosity method operates beyond the convergence results from Section III. In fact, the numerical results displayed in Fig. 3 show that adding viscosity only to the highest resolution level suffices to already stabilize the computed solution. This yields better accuracy away from the singularity, as less artificial diffusion is introduced into the original problem. However, an oscillation due to the Gibbs phenomenon concentrated around the singularity presents itself. Although its support decreases as we improve the resolution, this artifact remains for every $J$.

\section{B. Wavelets and Singularities}

The description and detection of singularities by wavelet analyses has been envisaged by numerous authors since their introduction in the late $80 \mathrm{~s}$. Typical results relate the local Hölder, Lipschitz, or Besov regularity of a function with the decay across scales of its local wavelet coordinates.

To assess the qualitative behavior to which one should compare the behavior of coefficients of the reconstructions in Fig. 3, we briefly provide an example of the representation of a discontinuous function in terms of the wavelet basis that we are using. This will help us to disentangle the effects of the discontinuity from the effects of the Gibbs phenomenon by inspecting the coefficients in the proximity of the jump at $x=0.5$.
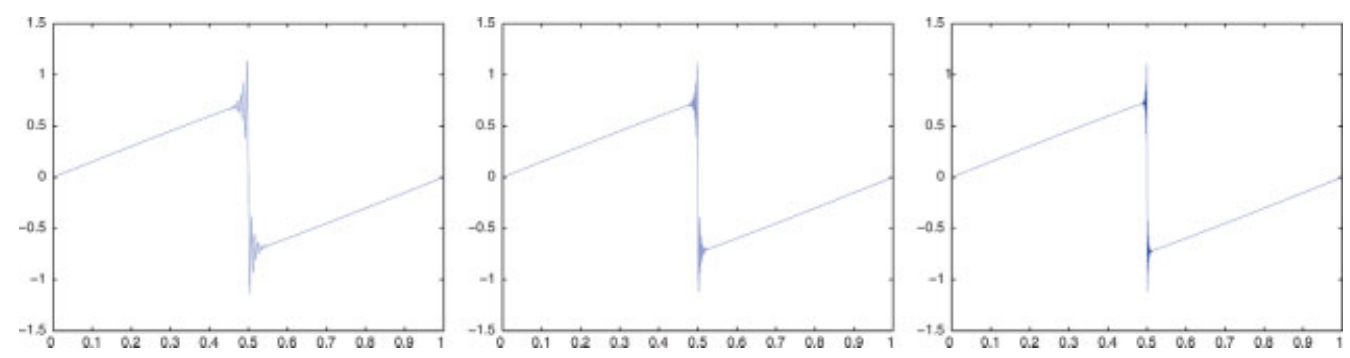

FIG. 3. Wavelet solutions for the stationary shock problem (4.1) using different resolutions. Artificial viscosity is applied only at the maximal resolution level: from left to right, $j_{\text {cut }}=8$ for $J=8 ; j_{\text {cut }}=9$ for $J=9$ and $j_{\text {cut }}=10$ for $J=10$. [Color figure can be viewed in the online issue, which is available at www.interscience.wiley.com.] 

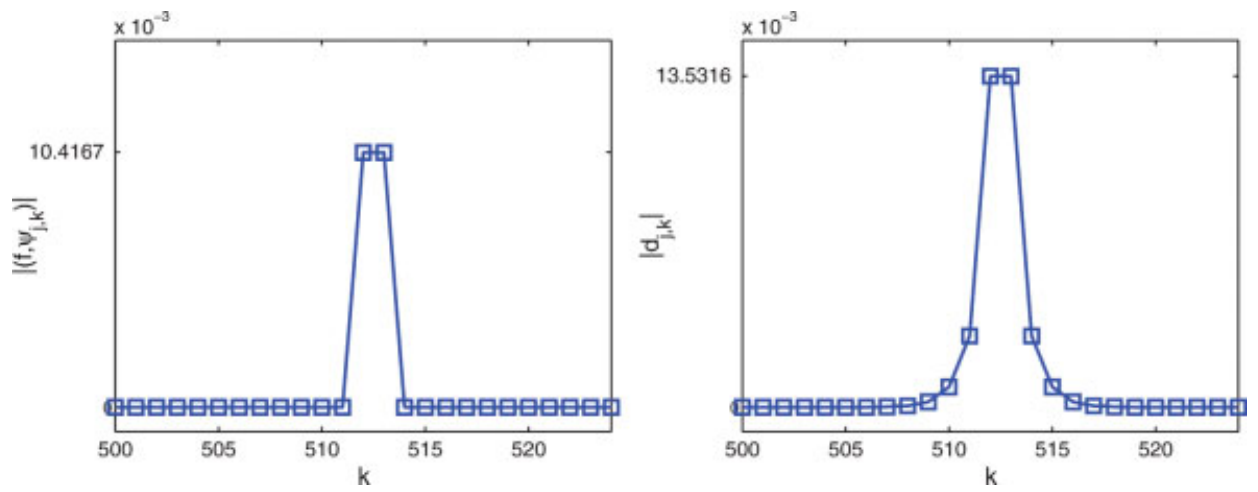

FIG. 4. Behavior of the wavelet coefficients of a given level ( $j=10$ ) across a discontinuity for the function $f(x)$ given in (4.1). Left: behavior of $\left|\int_{0}^{1} f(x) \psi_{j, k}(x) d x\right|$ against $k$ at level $j=10$. Right: behavior of $\left|d_{j, k}\right|$ against $k$ at level $j=10$. [Color figure can be viewed in the online issue, which is available at www.interscience.wiley.com.]

To study the decay profile of wavelet coefficients across a singularity, let $f$ be a linear function having a single jump discontinuity located at $x_{0}=1 / 2$, i.e.,

$$
f(x)=\left\{\begin{array}{cc}
x, & x \leq x_{0} \\
x-1, & x>x_{0}
\end{array}\right.
$$

We denote its wavelet expansion by

$$
f(x)=\sum_{j \geq j_{0} ; k=0, \ldots, 2^{j}-1} d_{j, k} \psi_{j, k}(x) .
$$

In view of the preorthogonal spline-wavelets employed here, the $L_{2}(0,1)$ scalar product of this function with all wavelets from level $j$ vanish on each level except for the two wavelets whose supports include the point $x_{0}=1 / 2$; see the left plot in Fig. 4 for the case $j=10$. Taking scalar products in $L_{2}$ of (4.1) with each wavelet on level $j^{\prime}$, we obtain

$$
\left(f, \psi_{j^{\prime}, k^{\prime}}\right)_{L_{2}(0,1)}=\sum_{j \geq j_{0} ; k=0, \ldots, 2^{j}-1} d_{j, k}\left(\psi_{j, k}, \psi_{j^{\prime}, k^{\prime}}\right)_{L_{2}(0,1)}, \quad k^{\prime}=0, \ldots, 2^{j^{\prime}}-1 .
$$

One can now solve for the wavelet coefficients $\left\{d_{j^{\prime}, k}\right\}_{k=0, \ldots, 2^{j^{\prime}}-1}$ of the expansion in (4.2) and obtain the right plot in Fig. 4. Note that multiplication of the wavelet coefficients by the entries of the mass matrix $\left(\psi_{j, k}, \psi_{j^{\prime}, k^{\prime}}\right)_{L_{2}(0,1)}$ introduces a smooth decay.

\section{Postprocessing}

An elementary smoothing technique applied for the reduction of Gibbs artifacts on the basis of (preorthogonal spline-)wavelets is described as follows: identify the spatial areas in which this phenomenon is active, eliminate the responsible wavelet coefficients, and recompute the remaining ones. This identification is based on the inspection of the decay of the wavelet coefficients at each scale, as represented in Fig. 5. The shape of the decay plot of the wavelet coefficients $\left|d_{j, k}\right|$ 

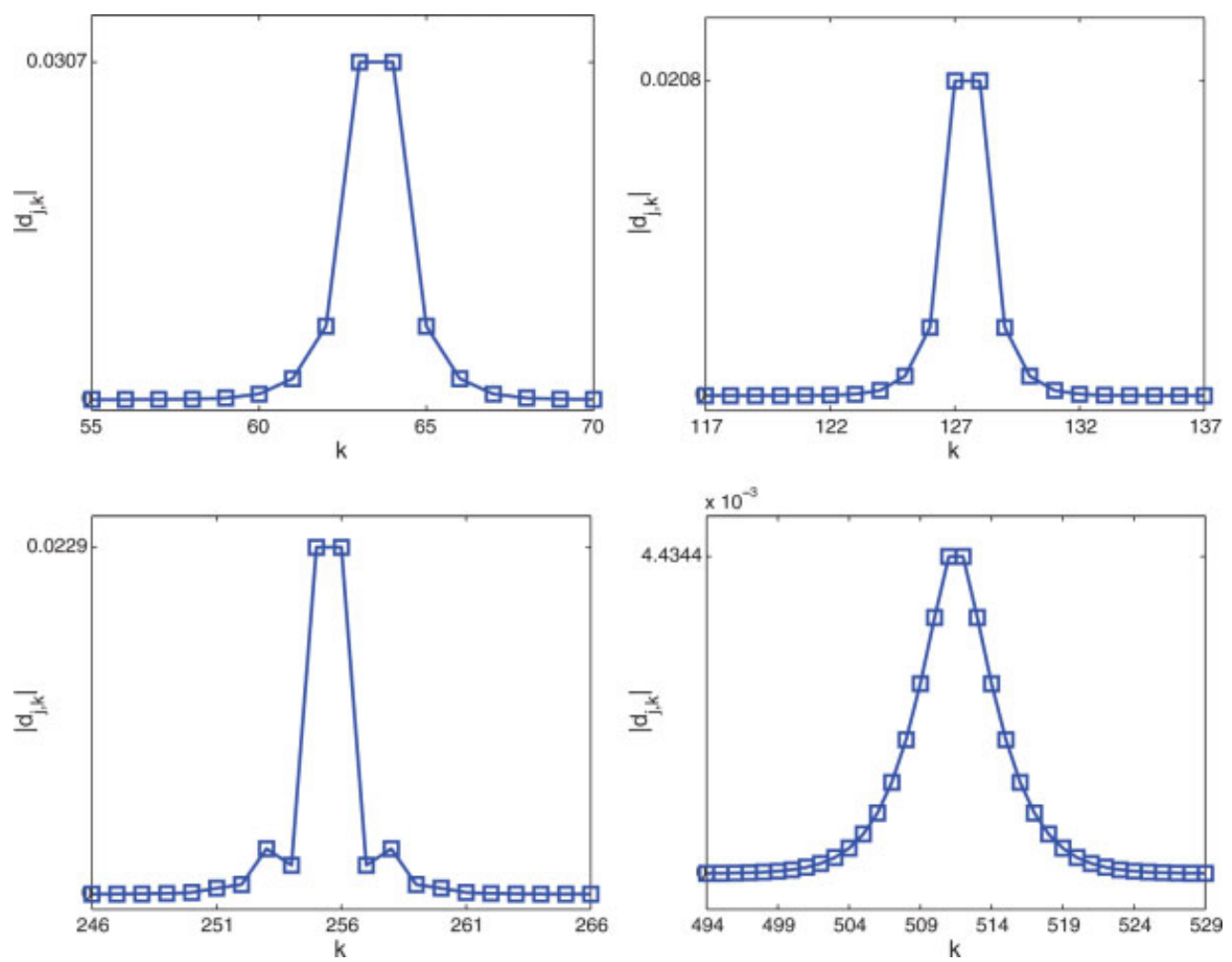

FIG. 5. Decay of wavelet coefficients on the last four levels before any smoothing technique is applied; $j=7,8,9,10$ from left to right. [Color figure can be viewed in the online issue, which is available at www.interscience.wiley.com.]

vs. $k$ for levels $j=7, j=8$, and $j=9$ is similar to the decay shown by the wavelet coefficients representing the discontinuous function $f(x)$ defined in (4.1) that has a similar singularity. In contrast to this, the decay of $\left|d_{10, k}\right|$ at level $j=10$ across $k$ is qualitatively different: since it is too wide, it does not match the decay expected from a "clean" discontinuity as seen in the example from Section B.

Let us denote by $\Lambda_{\text {Gibbs }}$ the set of indices of wavelets whose coefficients appear to be affected by the Gibbs phenomenon. The decay across $k$ of the wavelet coefficients signals an energy concentration along an extended interval

$$
I_{\text {Gibbs }}:=\bigcup_{\lambda \in \Lambda_{\text {Gibbs }}} \operatorname{supp} \psi_{\lambda}
$$

We assume that this interval marks approximately the support of the artifact and that the reconstruction on this interval is henceforth of no use. Now, one simply performs a local smooth re-sampling of the signal using only points lying outside $I_{\text {Gibbs }}$ and computes the wavelet coefficients with respect to this new resampling. A possible choice is $X_{\Lambda}^{(m)} / X_{\Lambda_{\text {Gibbs }}^{(m)}}^{\text {for any } m>0, \Lambda}$ being the original wavelet configuration. The right plot in Fig. 6 shows the reconstruction attained by this elementary technique for the solution of highest resolution in Figure 3. Figure 7 shows that this strategy yields different results from those obtained by simply discarding the coefficients of the higher, offending scales: the coefficients on levels $j=9,10$ do indeed disappear (and 

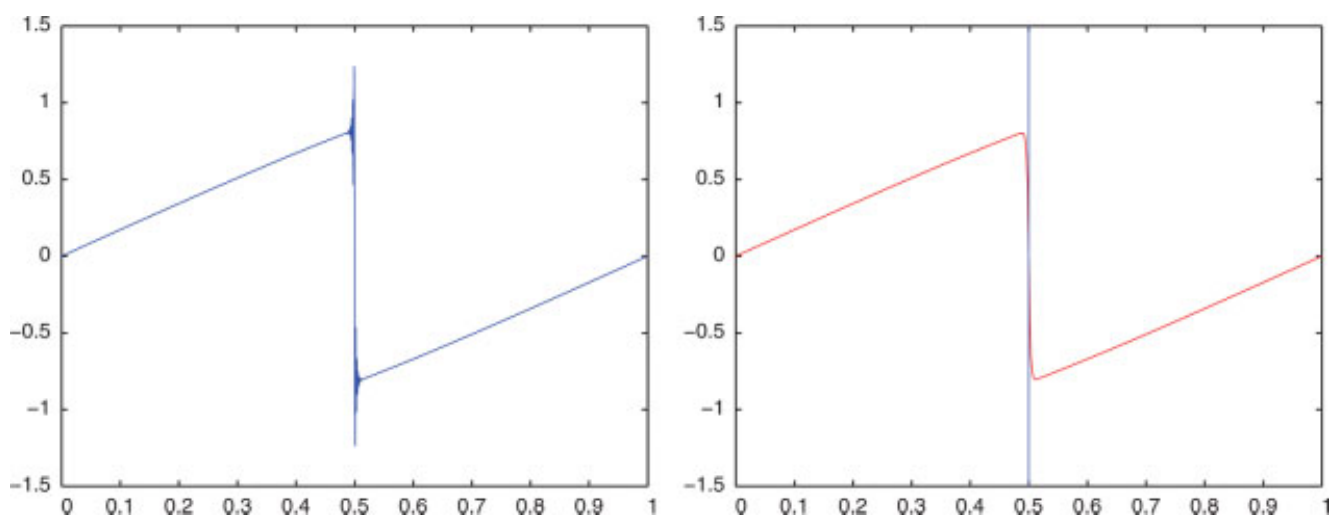

FIG. 6. Smoothing the approximate solution for level $J=10$ for the stationary shock (4.1); left: without smoothing; right: with smoothing. [Color figure can be viewed in the online issue, which is available at www.interscience.wiley.com.]

they are not depicted), but also the coefficients of the remaining levels are changed. In fact, if one just threw out wavelet coefficients corresponding to the higher scales, one would just end up rescaling the Gibbs phenomenon and getting a wider $I_{\text {Gibbs }}$; see Fig. 3. Thus, we see that the Gibbs phenomenon is not located exclusively on the two upper levels $(j=9,10)$. It leaks down through the lower scales. The correction is therefore stronger for higher levels and fades away as we move down in the dyadic scale of resolution; compare the plots for $j=5$ and $j=8$.

Obviously, this procedure decreases the sharpness of the peak: the final resolution in the example is $j=8$ after postprocessing. This does not mean, however, that the work done to resolve the discontinuity at level $j=10$ is lost. In fact, if we started with a signal resolved at level $j=8$, the smoothing would not take into account the information coming from the two last levels and we would just produce a rescaled version of the Gibbs phenomenon.

Starting with a given finest level $j$, if one wishes to suppress the artifacts and simultaneously wishes to keep a sharp resolution of the discontinuity up to some level, one would need more involved techniques. Among these are powerful strategies drawn from the image processing community such as the minimization of the total variation $[19,20]$ and the footprint location method [21] that are aimed at squeezing out the last drop of information from the given set of available points. In the present context, one could gain additional information by producing solutions of higher resolution by locally adding degrees of freedom; we will consider this approach in Section V.

The postprocessing technique carries over to singularities arising in other situations. The following problem develops a sharp shock that travels from left to right:

$$
\begin{aligned}
\frac{\partial u}{\partial t}+\frac{\partial}{\partial t}\left(\frac{u^{2}}{2}\right) & =0 & & \text { on }(0,1) \times(0, \\
u(0, t) & =u(1, t) & & \text { for all } t \in(0, T) \\
u(x, 0) & =1+\frac{1}{2} \sin (\pi(2 x-1)) & & \text { for } x \in(0,1) .
\end{aligned}
$$

This example was used in [3]; its exact solution is implicitly given by solving a nonlinear equation [5]. The numerical solution is computed here with (preorthogonal spline-)wavelets with respect to a uniform discretization and the explicit Euler method for time discretization that 

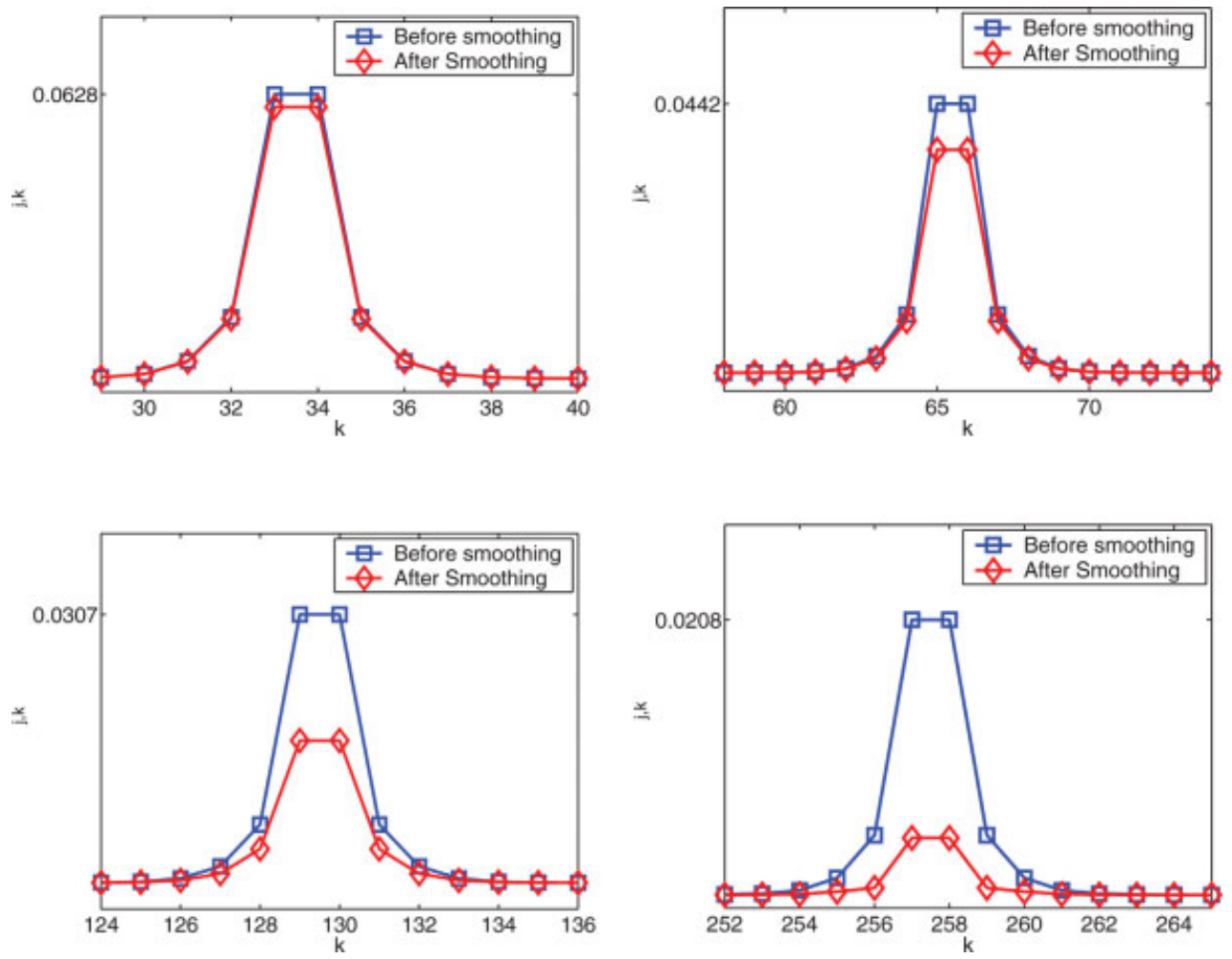

FIG. 7. Decay of the coefficients on the last four levels after smoothing compared to their original values; $j=5,6,7,8$ from left to right. [Color figure can be viewed in the online issue, which is available at www.interscience.wiley.com.]

yields qualitatively the same results as more involved schemes provided that the time step is small enough.

Figure 8 shows the instabilities that appear in absence of artificial viscosity. The results at time $t=0.5$ using artificial diffusion on only finest level are displayed in Fig. 9 for different values of $J$, illustrating the convergence of the method as the resolution level increases.

\section{AN ADAPTIVE WAVELET VISCOSITY SCHEME}

Although the convergence results of Section III apply for a full-grid discretization, we have found that spatially adaptive schemes also benefit from the frequency-selective addition of artificial viscosity. We have, in fact, implemented a slight modification of the adaptive algorithm proposed in [8]. The approximation of the solution $u(m \delta t, \cdot)$ at time $m \delta t$ is given by $u^{m}=\sum_{\lambda \in \Lambda^{m}} d_{\lambda}^{m} \psi_{\lambda}$. The configuration $\Lambda^{m}$ is updated by a dynamically adaptive scheme, and the time evolution is computed by a second-order Adams-Bashforth scheme.

The selection of indices that participate in the refinement step proceeds in two sequential stages. First, since the configuration at the next time step may require wavelets of a higher level, one adds all children of the currently chosen wavelet indices. Recall that in one spatial dimension, a wavelet indexed by $(j, k)$ is called a child of a wavelet indexed by $\left(j^{\prime}, k^{\prime}\right)$ if $j=j^{\prime}+1$ and $k \in\left\{2 k^{\prime}, 2 k^{\prime}+1\right\}$, and correspondingly in the multivariate case. In the second stage, this 


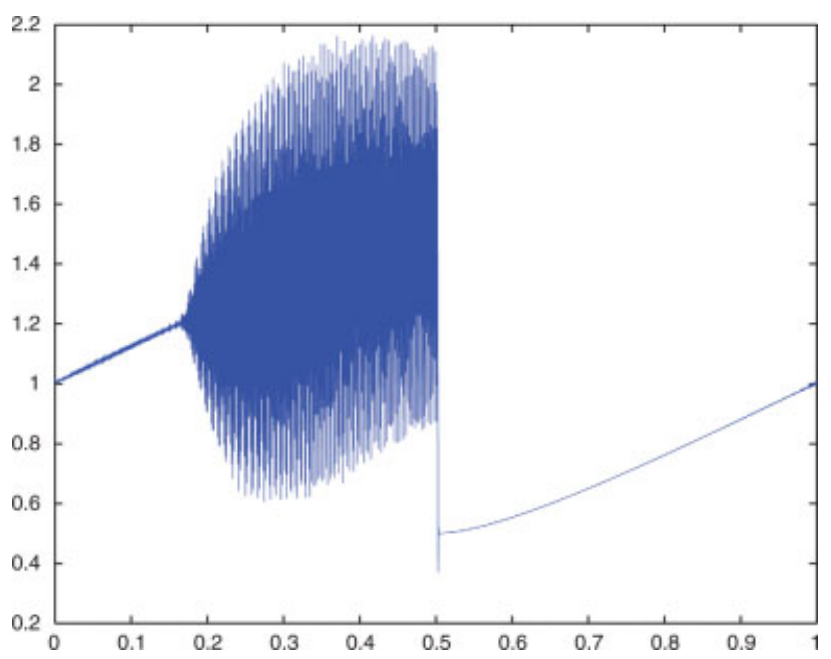

FIG. 8. Instabilities arising at time $T=0.5$ in the absence of artificial viscosity when solving the traveling shock problem for $J=10$. [Color figure can be viewed in the online issue, which is available at www.interscience.wiley.com.]

index set is further extended by also including the horizontal neighbors of the wavelet indices already included. We then define the following algorithm that includes a coarsening step based on thresholding the latest available wavelet coefficients.

Algorithm 5.1. [Adaptive Wavelet-Galerkin Scheme] Fix a threshold $\varepsilon(j)$ dependent on the resolution level. Given an initial solution $u^{0}$ expanded in terms of wavelets relative to some index
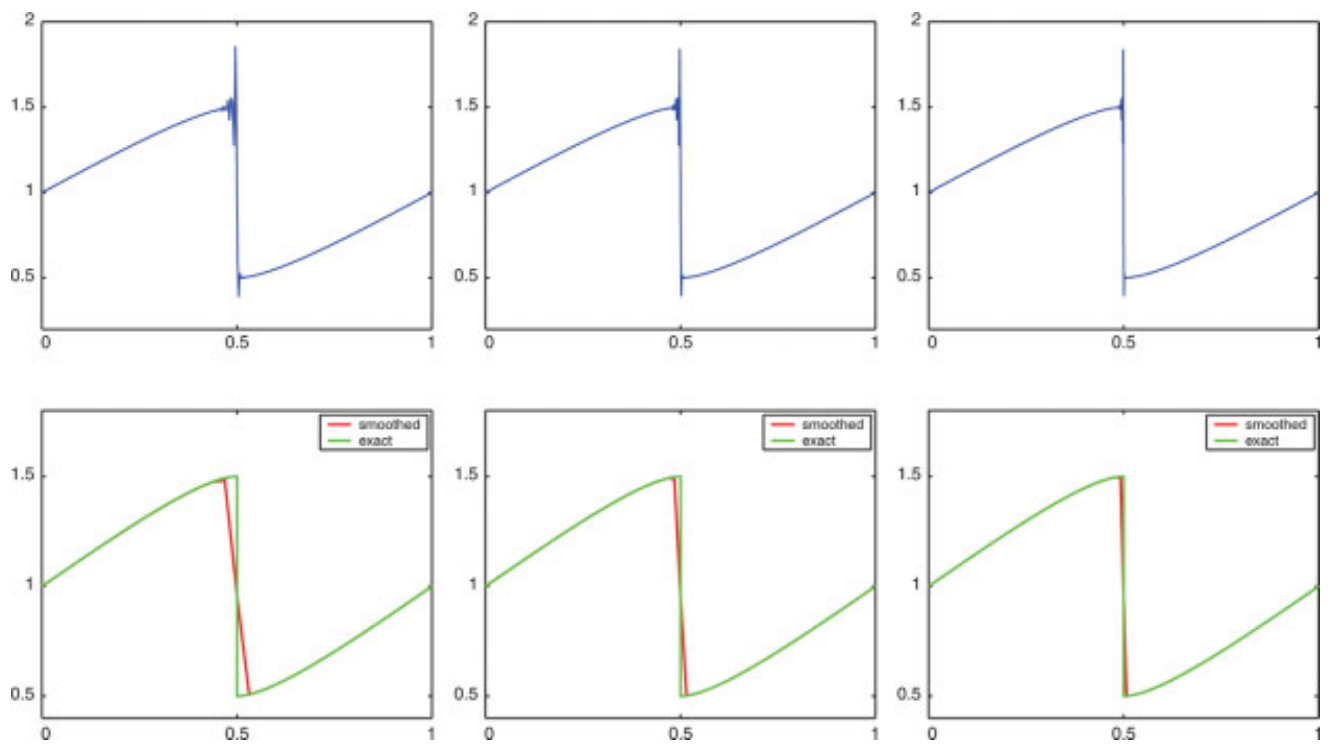

FIG. 9. Wavelet solution with the full grid for the traveling shock problem. The upper row shows the results at $T=0.5$ for different resolution levels $j=8,9,10$ from left to right. The lower row shows the results after smoothing, compared to the exact solution. [Color figure can be viewed in the online issue, which is available at www.interscience.wiley.com.] 
set $\widetilde{\Lambda}^{0}$, define $\Lambda^{0}$ by thresholding $\widetilde{\Lambda}^{0}$, i.e. $\Lambda^{0}:=\left\{\lambda \in \widetilde{\Lambda}^{0}:\left|d_{\lambda}\right|>\varepsilon(0)\right\}$. Then, for each $m=0, \ldots$, perform the following steps.

\section{(1) Refinement}

(a) Vertical Expansion: construct a set $\Lambda_{v}^{m}$ containing the wavelet indices in $\Lambda^{m}$ and all their children.

(b) Horizontal Expansion: form

$$
\widetilde{\Lambda}^{m+1}:=\left\{\lambda=(j, k) \text { such that }\left(j, k^{\prime}\right) \in \Lambda_{v}^{m}, k^{\prime} \in\{k-2, k-1, k, k+1, k+2\}\right\} .
$$

(2) Computation of approximate solution: compute

$$
u^{m+1}=\sum_{\lambda \in \widetilde{\Lambda}^{m+1}} d_{\lambda}^{m+1} \psi_{\lambda}
$$

that solves

$$
\left(\frac{1}{\tau}\left(u^{m+1}-u^{m}\right)+\frac{1}{2} \frac{\partial}{\partial x}\left(u_{*}^{m}\right)^{2}, \psi_{\lambda}\right)=-v\left(\frac{\partial}{\partial x} Q_{J}\left(u_{*}^{m}\right), \frac{\partial}{\partial x} \psi_{\lambda}\right) \text { for } \lambda \in \widetilde{\Lambda}^{m+1},
$$

with $u_{*}^{m}=\frac{3}{2} u^{m}-\frac{1}{2} u^{m-1}$ (with $u_{*}^{m}=u^{m}$ for $m=0$ ).

(3) Coarsening: compute $\Lambda^{m+1}$ by thresholding $\widetilde{\Lambda}^{m+1}$ to obtain

$$
\Lambda^{m+1}:=\left\{\lambda \in \widetilde{\Lambda}^{m+1}:\left|d_{\lambda}\right|>\varepsilon(|\lambda|)\right\}
$$

To illustrate the performance of this algorithm, we have solved Burgers' equation for the two different initial conditions introduced in Section IV. In both cases, the following parameter set has been used: $J_{\max }=10, v=2^{-\left(J_{\max }-1\right)}, \varepsilon(j)=10^{-6} \cdot 2^{-j}$. The artificial viscosity acts only on the elements with $j>6$. The results are shown in Fig. 10 for the stationary shock problem and in Fig. 11 for the traveling shock problem. In the top rows, the configuration of the wavelet coefficients is displayed, exhibiting their spatial distribution over the $x$-axis. The vertical axis shows the
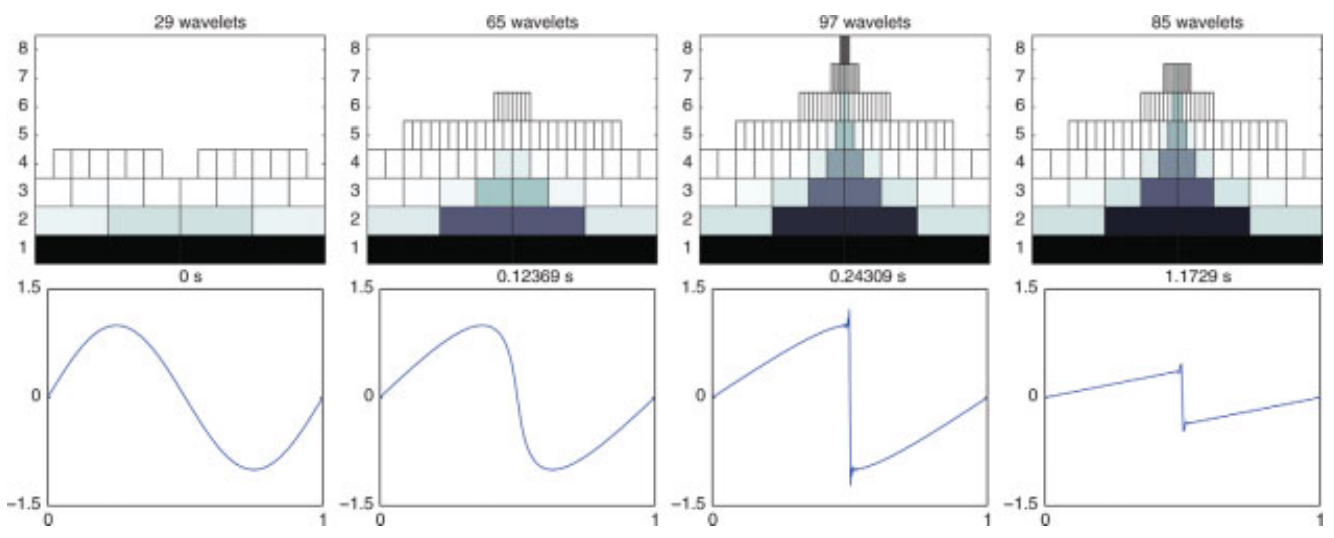

FIG. 10. Top row: evolution of the wavelet configuration selected by the adaptive method applied to the stationary shock problem (4.1) for $t=0,0.12,0.24,1.17$ from left to right. Bottom row: the corresponding approximate solutions. [Color figure can be viewed in the online issue, which is available at www.interscience.wiley.com.] 

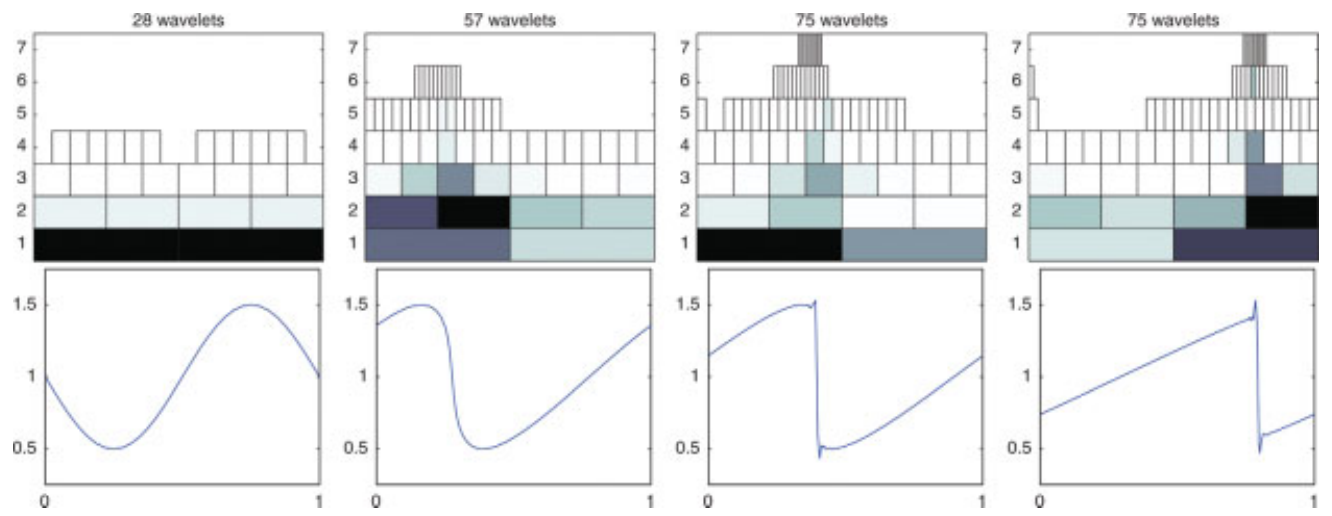

FIG. 11. Top row: evolution of the wavelet configuration selected by the adaptive method applied to the traveling shock problem (4.4) for times $t=0,0.28,0.39,0.78$ from left to right. Bottom row: the corresponding approximate solutions. [Color figure can be viewed in the online issue, which is available at www.interscience.wiley.com.]

refinement level $j$ with increasing value from bottom to top. Darker colors of the boxes refer to higher absolute and, therefore, more important values of the corresponding wavelet coefficients. To compare with the solutions on the full uniform grid displayed in Section IV, no postprocessing has been applied.

We see that in both cases the scheme appears to perfectly capture the dynamics of the solution. The initial wavelet structure expands itself to resolve the forming shock as the method selects wavelets in the neighborhood of the developing discontinuity. The prescribed thresholding policy prevents the method from accumulating wavelets of resolution higher than level $j=8$. For the stationary shock example, the discontinuity remains fixed at $x=0.5$ and reduces in size, leading to a simplification of the wavelet structure as time increases. For the case of the traveling shock, the wavelet structure travels with the shock. These examples show that numerical instabilities do indeed disappear.

\section{EVALUATION OF NONLINEARITIES}

Up to this point, we have not specified how the nonlinear term in (5.2) is computed in terms of adaptive spline-wavelets. To this end, we have employed a least-squares approach to evaluate nonlinearities described in [10] for which the Burgers' problem provides an interesting benchmark.

The task at hand is to evaluate nonlinear terms of the form $\left\{\left\langle\psi_{\lambda}, G(u)\right\rangle\right\}_{\lambda \in \Lambda}$, where $u$ is a known function which can be expressed as a finite linear combination of wavelets, or, equivalently, as a vector $\mathbf{u}$, with $\Lambda$ being a given set of wavelet indices. If the wavelet representation of $G(u)=\sum_{\lambda \in I} g_{\lambda} \psi_{\lambda}$, or $\mathbf{g}:=\left\{g_{\lambda}\right\}_{\lambda \in I I}$ in vector form, is known, this would allow for the evaluation of $\mathbf{G}(\mathbf{u})$ exactly by noting that

$$
\int_{0}^{1} G(u(x)) \psi_{\lambda}(x) d x=\sum_{\lambda^{\prime} \in I} g_{\lambda^{\prime}} \int_{0}^{1} \psi_{\lambda^{\prime}}(x) \psi_{\lambda}(x) d x=\sum_{\lambda^{\prime} \in I} g_{\lambda^{\prime}} M_{\lambda, \lambda^{\prime}},
$$


where $M_{\lambda, \lambda^{\prime}}$ are the entries of the mass matrix M. Then, we would simply solve

$$
\mathbf{G}(\mathbf{u})=\mathbf{M g}
$$

for $\mathbf{g}$. In principle, infinitely many wavelets are needed to represent $G(u)$ exactly. However, we are interested only in those wavelets that are not orthogonal, with respect to $L_{2}$, to the wavelets indexed by the given set $\Lambda$, as only they can contribute to (6.1). Thus, we can work with a maximal set of indexes $\bar{\Lambda}:=\Lambda \cup \partial \Lambda$. Here, $\partial \Lambda$ stands for the set of indices of those wavelets that are not orthogonal to the ones indexed by $\Lambda$. For preorthogonal wavelets in $L_{2}, \partial \Lambda$ includes just the neighbors on the same resolution level of the wavelets in $\Lambda$, i.e.,

$$
\partial \Lambda=\left\{\lambda \in I I: \exists \lambda^{\prime} \in \Lambda \text { such that }|\lambda|=\left|\lambda^{\prime}\right|, \quad \operatorname{supp} \psi_{\lambda} \cap \operatorname{supp} \psi_{\lambda^{\prime}} \neq \emptyset\right\} .
$$

Note that $\bar{\Lambda}$ is finite if $\Lambda$ is.

Next, we construct an approximation to $\mathbf{g}_{\bar{\Lambda}}$ by means of a least-squares technique: we select some appropriate set of points $\widehat{X}$, evaluate $G\left(u\left(x_{i}\right)\right)$ at each $x_{i} \in \widehat{X}$ and use the adaptive Coarseto-Fine-Algorithm developed in [10] to obtain a configuration $\widehat{\Lambda} \subseteq \bar{\Lambda}$ and the coefficients $\left\{\widehat{g}_{\lambda}\right\}_{\lambda \in \widehat{\Lambda}}$ of the approximation $\widehat{g}(x):=\sum_{\lambda \in \widehat{\Lambda}} \widehat{g}_{\lambda} \psi_{\lambda}(x)$. The question of how to select the points $\widehat{X}$ in an appropriate and constructive way to guarantee an efficient and fast iterative solution of the least-squares problem has been answered in [10]: roughly, one needs a small fixed number of evaluation points $\widehat{m}$ in each subinterval, depending on the degree of polynomial making up the spline-wavelet $\psi_{\lambda}$.

Denoting by $\delta(\Lambda)$ the children of the highest-level-wavelets of a set $\Lambda$, our algorithm to evaluate nonlinearities reads as follows.

\section{Algorithm 6.1. [Least Squares Scheme for Evaluating Nonlinearities]}

1. Fix a maximal configuration $\bar{\Lambda}$, the coarsest level $j_{0}$, the order $\widehat{m}$ of the point representations, and a thresholding parameter $\varepsilon>0$. Create $\widehat{\Lambda}_{j_{0}}$.

2. For each $j=j_{0}, \ldots$, perform the following steps.

(a) Create $\delta\left(\widehat{\Lambda}_{j}\right)$.

(b) Construct $\widetilde{\Lambda}_{j+1}:=\widehat{\Lambda}_{j} \cup\left(\delta\left(\widehat{\Lambda}_{j}\right) \cap \bar{\Lambda}\right)$.

(c) Compute $\left\{\widehat{g}_{\lambda}^{j+1}\right\}_{\lambda \in \tilde{\Lambda}_{j+1}}$ that minimizes

$$
\sum_{x_{i} \in X_{\tilde{\Lambda}_{j+1}^{m}}^{m}}\left(G\left(u\left(x_{i}\right)\right)-\sum_{\lambda \in \tilde{\Lambda}_{j+1}} \widehat{g}_{\lambda}^{j+1} \psi_{\lambda}\left(x_{i}\right)\right)^{2} .
$$

(d) Select $\Lambda_{j+1}^{\varepsilon}:=\left\{\lambda \in \delta\left(\widehat{\Lambda}_{j}\right) \cap \bar{\Lambda}:\left|\widehat{g}_{\lambda}^{j+1}\right| \geq \varepsilon\right\}$.

(e) If $\Lambda_{j+1}^{\varepsilon}=\emptyset$ stop, else let $\widehat{\Lambda}_{j+1}=\widehat{\Lambda}_{j} \cup \Lambda_{j+1}^{\varepsilon}$.

Note that this approach differs from the one in $[22,23]$. In the above least-squares approach, the construction of the wavelet configuration $\widehat{\Lambda}$ and the computation of the corresponding coefficients $\left\{\widehat{g}_{\lambda}\right\}$ occur simultaneously: it creates a hierarchy of configurations $\widehat{\Lambda}_{j_{0}} \subset \widehat{\Lambda}_{j_{0}+1} \subset \ldots \subseteq \widehat{\Lambda}$ by using the coefficients on each level $j$ to predict the significant ones on the next level $j+1$. The use of the information about $G(u)$ gained at each level might allow for a possible termination of the algorithm at some early level before having computed the full configuration $\bar{\Lambda}$ so that $\# \widehat{\Lambda}<\# \bar{\Lambda}$. 
Note that the size of the trial configuration $\bar{\Lambda}$ may be overestimated by the algorithms described in [23] since there $\bar{\Lambda}$ is guaranteed to be large enough to fulfill the approximation requirement, but is not guaranteed to contain the smallest possible number of parameters.

A correction of the algorithmic drawbacks of [23] and [22] has been envisaged recently in [24]. The strategy there operates in a sweep from fine-to-coarse scales and is therefore opposite, in this respect, to our least-squares approach, and also relies on reusing the information gained in the analysis of each level.

\section{References}

1. E. Tadmor, Convergence of spectral methods for nonlinear conservation laws, SIAM J Numer Anal 26 (1989), 30-44.

2. H. Yserentant, On the multilevel splitting of finite element spaces, Numer Math 49 (1986), 379-412.

3. M. Calhoun-Lopez and M. D. Gunzburger, A finite element, multiresolution viscosity method for hyperbolic conservation laws, SIAM J Numer Anal 43 (2005), 1988-2011.

4. M. Calhoun-Lopez and M. D. Gunzburger, The efficient implementation of a finite element, multiresolution viscosity method for hyperbolic conservation laws, J Comp Phys 225 (2007), 1288-1313.

5. M. Calhoun-Lopez, Numerical solutions of hyperbolic conservation laws: incorporating multi-resolution viscosity methods into the finite element framework, Ph.D. Thesis, Iowa State University, 2003.

6. W. Layton, A connection between subgrid-scale eddy viscosity and mixed methods, Appl Math Comput 133 (2002), 147-157.

7. V. John and S. Kaya, A finite element variational multiscale method for the Navier-Stokes equations, SIAM J Sci Comput 26 (2005), 1485-1503.

8. P. Joly, Y. Maday, and V. Perrier, A dynamical adaptive concept based on wavelet packet best bases: application to convection diffusion partial differential equations, W. Dahmen, A. Kurdila, and P. Oswald, editors, Multiscale wavelet for partial differential equations, Academic Press, New York, 1997, pp. 199-236.

9. J. Regele and O. Vasilyev, An adaptive wavelet-collocation method for shock computations, J Comput Phys, submitted.

10. D. Castaño Díez, Adaptive scattered data fitting with tensor product spline-wavelets, Ph.D. Dissertation, Universität Bonn, January, Available at: http://hss.ulb.uni-bonn.de/diss_online/ math_nat_fak/2005/castano-diez_daniel, 2005.

11. W. Dahmen, Wavelet and multiscale methods for operator equations, Acta Numerica (1997), 55-228.

12. A. Kunoth, Wavelet methods - boundary value problems and control problems, Advances in Numerical Mathematics, Teubner, Wiesbaden, Germany, 2001.

13. A. Cohen, W. Dahmen, and R. DeVore, Adaptive wavelet methods for elliptic operator equations convergence rates, Math Comput 70 (2001), 27-75.

14. A. Cohen, W. Dahmen, and R. DeVore, Adaptive wavelet scheme for nonlinear variational problems with convergence rates, SIAM J Numer Anal 41 (2003), 1785-1823.

15. W. Dahmen, Multiscale and wavelet methods for operator equations, C.I.M.E. lecture notes, SpringerVerlag, Berlin 2003.

16. A. Kunoth and J. Sahner, Wavelets on manifolds: an optimized construction, Math Comp 75 (2006), 1319-1349.

17. E. Stollnitz, T. DeRose, and D. Salesin, Wavelets for computer graphics, Morgan Kaufmann Publishers, San Francisco, 2000. 
18. C. Chui and E. Quak, Wavelets on a bounded interval, D. Braess and L. L. Schumaker, editors, Numerical Methods of Approximation Theory, Vol. 9, Birkhäuser, Basel, 1992, pp. 53-75. International Series of Numerical Mathematics 105.

19. S. Durand and J. Froment, Artifact free signal denoising with wavelets, Proc. of ICASSP'01, vol. 6, 2001.

20. S. Durand and J. Froment, Reconstruction of wavelet coefficients using total variation minimization, SIAM J Sci Comput 24 (2003), 1754-1767.

21. P. Dragotti and M. Vetterli, Wavelet footprints: theory, algorithms and applications, IEEE Trans Signal Process 51 (2003), 1306-1323.

22. A. Cohen, W. Dahmen, and R. DeVore, Sparse evaluation of compositions of functions using multiscale expansions, SIAM J Math Anal 35 (2003), 279-303.

23. W. Dahmen, R. Schneider, and Y. Xu, Nonlinear functionals of wavelet expansions-Adaptive reconstruction and fast evaluation, Numer Math 86 (2000), 49-101.

24. A. Barinka, W. Dahmen, and R. Schneider, Fast computation of adaptive wavelet expansions, Numer Math 105 (2007), 549-589. 